\title{
La pareja y su terapeuta. Conceptualizaciones, evoluciones y desafíos ${ }^{1}$ The couple and their therapist: Conceptualizations, evolutions and challenges
}

Deisy González Quiros ${ }^{*}$, Daniela Ramírez Gómez $^{* *}$, Cindy Sepúlveda Flórez ${ }^{* * *}$

Forma de citar este artículo en APA:

González Quiros, D., Ramírez Gómez, D., \& Sepúlveda Flórez, C. (2021). Aproximación al aprendizaje pedagógico en la discapacidad intelectual leve. Poiésis, (41), 174-190. https://doi.org/10.21501/16920945.4191

\section{Resumen}

El presente artículo se deriva de una investigación documental, la cual tuvo como objetivo analizar el concepto de pareja y las habilidades del terapeuta de pareja, que se identificaron en fuentes documentales de terapia contemporánea. En primer lugar, se logró identificar los cambios que se han dado en la conceptualización de la pareja, ya que la pareja como concepto ha tenido una fuerte implicación histórica y cultural, evidenciándose nuevas tipologías de pareja en la contemporaneidad. También fue posible encontrar que la pareja ha sido leída como sistema desde el trabajo terapéutico con la influencia de la cibernética de segundo orden. En segundo lugar, se hallaron algunas de las habilidades que un terapeuta de pareja debe desarrollar para el éxito de su intervención, entre las que se encuentran la identificación de motivo de consulta, el establecimiento de la alianza terapéutica y el desarrollo del self del terapeuta.

\section{Palabras clave:}

Alianza terapéutica; Concepto de pareja; Habilidades del terapeuta; Sistema; Terapeuta; Terapia, Tipologías de pareja.

Artículo derivado del proceso de investigación documental realizado para optar por el título de Especialistas en Terapia Familiar en la Universidad Católica Luis Amigó, Medellín (Colombia), bajo la asesoría de la Mg. Yeny Leydy Osorio Sánchez.

Trabajadora social, estudiante del programa de especialización de Terapia Familiar de la Universidad Católica Luis Amigó, MedellínColombia. Contacto: deisy.gonzalezqu@amigo.edu.co

Trabajadora social, estudiante del programa de especialización de Terapia Familiar de la Universidad Católica Luis Amigó, MedellínColombia. Contacto: daniela.ramirezgo@amigo.edu.co

Trabajadora social, estudiante del programa de especialización de Terapia Familiar de la Universidad Católica Luis Amigó, MedellínColombia. Contacto: cindy.sepulvedafl@amigo.edu.co 


\section{Abstract}

This article is derived from documentary research, which aimed to analyze the concept of the couple and the skills of the couple therapist, which were identified in documentary sources of contemporary therapy. In first place, it was possible to identify the changes that have occurred in the conceptualization of the couple, since the couple as a concept has had a strong historical and cultural implication, evidencing new types of couples in contemporary times. It was also possible to find that the couple has been read as a system from therapeutic work with the influence of second-order cybernetics. Second, some of the skills that a couples' therapist must develop to succeed in their intervention were found, among which are the identification of the reason for consultation, the establishment of the therapeutic alliance and the development of the therapist's self.

\section{Keywords:}

Therapeutic alliance; Concept of couple; Therapist's skills; System; Therapist; Therapy; Couple typologies. 


\section{Introducción}

La vida del ser humano está estructurada por una serie de experiencias y relaciones que dan cuenta de una historia personal de permanente interacción con el mundo y con el otro. Una de las relaciones humanas más complejas es la relación de pareja, la cual ha sufrido transformaciones a lo largo de la historia a partir de los cambios que tienen lugar en el mundo social.

Es así como el concepto de pareja ha sido dinámico, ha ido mutando, debido a que la pareja no solo consiste en dos personas en interacción con un fin de procreación, sino que ha sido concebida de acuerdo con los diferentes momentos históricos y las instituciones que han influenciado su actuar.

A pesar de que históricamente se ha concebido desde una perspectiva tradicional a la pareja, en la actualidad se aceptan formas de pareja que proponen nuevas sexualidades e identidades de género o que no siguen parámetros establecidos, por lo que es un reto vigente el hecho de reconocer la multiplicidad de formas en que es posible ser un sistema diádico.

De otro lado, debe considerarse que las parejas presentan problemáticas que generan dificultades y ponen en riesgo su estabilidad, lo que las convoca en ocasiones a buscar ayuda en la terapia de pareja, la cual se ha comprendido como el abordaje terapéutico que se realiza con la diada que presenta una queja o una dificultad relacional. De acuerdo con la teoría de sistemas, la pareja constituye un sistema y como tal es susceptible de ser intervenido. Agudelo y Palacio (2015), refieren que

desde la terapia sistémica, las problemáticas de la pareja no se conciben como derivadas únicamente de lo que hace uno u otro sino que se abarca la relación en la que cada quien hace 0 deja de hacer algo que suscita sentimientos o reacciones negativas recíprocas que no se enfrentan explícitamente si no que se encubren, se postergan y se agudizan, configurando problemáticas que dañan la relación y deterioran el vínculo. (p. 80)

No es un secreto que a lo largo de la historia se han dado al interior de la pareja algunas problemáticas como son: la infidelidad, dificultades con las familias de origen, periodos de distanciamiento entre los miembros de la diada, problemas relacionados a la sexualidad, desacuerdos en la crianza de los hijos, entre otros, situaciones que afectan la relación, tienden a perpetuarse e irrumpen con la dinámica familiar poniendo en juego la estabilidad de la pareja. Es a partir de estos desencuentros donde el terapeuta de pareja deberá centrar su mirada en las dinámicas de relacionamiento al interior del sistema. 
De acuerdo con Vicencio (2011), desde el enfoque sistémico procedente de la cibernética de segundo orden, durante la terapia se "permite explorar los significados específicos que cada pareja le otorga a su relación de acuerdo con las historias más significativas de cada uno de ellos o de ambos en sus experiencias amorosas conjuntas, previas o fantaseadas" (p. 3 ).

Es en este espacio terapéutico donde el rol del terapeuta se dota de sentido, en la medida en que se reconoce como un ser humano en interacción con los otros; las parejas que consultan a terapia se enfrentan a la situación de develar su historia, exponerse y desnudar su intimidad emocional. En el contexto de la terapia de pareja, entonces, el terapeuta

es un ser humano que se interesa terapéuticamente por sus prójimos en ámbitos y problemas que los hacen sufrir, al tiempo que observa un gran respeto por sus valores, sus intereses y sus preferencias estéticas. En otras palabras, la meta es trascender lo técnico. (Minuchin \& Fishman, 2004, p. 15)

El terapeuta desde esta lectura, no se comporta como un observador externo respecto de la pareja; por el contrario, "abre un espacio para la reflexión sobre el propio comportamiento (...). Dado que se fundamenta en la premisa de que no somos descubridores de un mundo exterior a nosotros, sino inventores o constructores de la propia realidad" (Foester, como se cita en Estrada et al., 1997, p. 38).

Teniendo en cuenta lo anterior se identifica la importancia de la realización de esta investigación documental, la cual estuvo guiada por la pregunta acerca de cómo ha sido abordado el concepto de pareja y las habilidades del terapeuta de pareja construyendo así dos categorías de análisis que permitieron orientar el proceso de rastreo documental, las cuales fueron el concepto de pareja y las habilidades del terapeuta.

Finalmente, de esta investigación se permite concluir que el concepto de pareja al ser una construcción social es una noción inacabada y requerirá de constante investigación; por consiguiente, el terapeuta debe desarrollar habilidades que estén en permanente construcción y mantener la curiosidad, con el fin de hacer conscientes las prácticas terapéuticas, formativas e investigativas.

\section{Metodología}

Esta investigación se realizó bajo el enfoque cualitativo y el método hermenéutico, a través de una revisión de tipo documental, que parte de comprender e interpretar realidades complejas desde fuentes de investigación y teóricas existentes. Se hizo una búsqueda y análisis de documentos relacionados con el concepto de pareja, habilidades del terapeuta de pareja y terapia de pareja. El estudio tuvo un alcance descriptivo y analítico; descriptivo porque se explicaron los hallazgos dando a conocer los detalles que aportan al desarrollo de los objetivos, y analítico porque a partir de estos documentos se logró realizar una revisión minuciosa y razonada de las posturas plasmadas por los autores. 
Para dar cuenta de este proceso, se realizó una exploración de información a partir de la depuración de fuentes documentales, realizando un análisis y filtrando de acuerdo con los criterios de inclusión y exclusión acordados. Se efectuó la selección de documentos extraídos en bases de datos o motores de búsqueda tales como Scielo, Pubmed, Redalyc, Research Gate, documentos que figuran en Google Académico, entre otros; teniendo en cuenta que los documentos fuesen en idioma español y que estuvieran directa o indirectamente relacionados con el tema; a su vez se incluyeron todos los artículos publicados sin importar país de origen, artículos de investigación, revisión de casos, entre otros, que han sido publicados entre 2000 al 2019.

Los términos clave, para realizar la revisión fueron "pareja", "terapeuta de pareja" y "terapia de pareja", y se le enlazaron con palabras como: concepto, historia, sistema, características, intervención, rol, función, estrategias y definición. Estos elementos de búsqueda posteriormente permitieron realizar la clasificación y el análisis de las categorías objeto de investigación. De este proceso se derivaron algunos otros conceptos que necesariamente fueron clave para el análisis, entre ellos, vínculo amoroso, tipologías de pareja, contrato de la diada, habilidades terapéuticas, alianza terapéutica y self del terapeuta.

Hecho el primer filtro a partir de los motores de búsqueda, se determinaron cuarenta artículos científicos a los cuales se les realizó una revisión a partir de la ficha de contenido, que abarcaba aspectos como: título, origen, autor, año, link, palabras clave, resumen y citas textuales o conceptos relevantes.

Finalmente, se utilizó el árbol de argumentos, metodología que permite mediante el análisis documental la construcción de un hallazgo central o conclusión global, desligándose de allí diversos argumentos o hallazgos secundarios que fueron soportados cada uno con evidencias bibliográficas.

\section{Resultados}

Para el análisis en esta investigación documental se tomaron como categorías clave el concepto de pareja y las habilidades del terapeuta. Ambas categorías permitieron comprender la relación y las implicaciones de la pareja como sistema y de la persona del terapeuta para el ejercicio del proceso terapéutico. A continuación, se desarrolla lo que corresponde a cada una de estas categorías.

\section{Concepto de pareja}

Las fuentes consultadas mostraron que la pareja es un tema de interés teórico e histórico y se ha hablado de la misma en las diferentes culturas y épocas; es por esto que la literatura muestra que el concepto de pareja tiene una fuerte influencia social y que ha ido transformándose a través del tiempo. A esta mirada histórica y social se suma un análisis sistémico desde el cual se asume que la pareja se conforma como un sistema desde el espacio relacional y que tiene como característica la circularidad. 
Detengámonos, inicialmente, en la perspectiva histórica cultural. Las uniones de pareja en épocas pasadas eran reguladas por la religión y se caracterizaban por la presencia de acuerdos, como un tipo de negociación o transacción con intereses de tipo económico de las familias o por estatus social, este tipo de uniones se identificaron por tener unos fines reproductivos donde la sexualidad no tenía como fin último el placer.

Lo anterior se evidencia en lo que señalan Garrido et al. (2008): "antes la pareja se elegía con base en los recursos económicos, étnicos o el interés político de las familias" (p. 232). En esta misma vía Eguiluz (2007) señala que en las antiguas civilizaciones las creencias religiosas permeaban la constitución de las parejas, en las cuales la castidad era un requisito para los esposos, quienes se unían solo con el fin de la procreación.

Esta forma de ser pareja comienza a cambiar a partir de la Revolución Francesa, periodo en el que el vínculo amoroso comienza a tomar validez, por ende, las uniones eran acordadas por ambos miembros de la diada. En 1792 surge por primera vez el divorcio, aunque luego es abolido en 1816. De acuerdo a lo revisado en este estudio, las parejas empezaban a dar pequeños pasos para el disfrute de la sexualidad; sin embargo, las uniones seguían teniendo como objetivo principal la reproducción, es decir, las parejas tenían como condicionante tener hijos para formar una familia (Eguiluz, 2007).

Es solo hasta el siglo XX cuando se empieza a gestar un nuevo concepto de pareja, uno que apela a otros tipos de unión sin estar necesariamente atravesados por parámetros religiosos y que implican el estar casados o tener hijos; así mismo surge el control de la natalidad y retoma con mayor fuerza la idea del divorcio y la separación.

Este cambio de concepción estuvo atravesado por diferentes movimientos culturales, entre ellos aquellos que condujeron a la formalización de los derechos de la mujer. Con respecto a esto se manifiestan varios de los autores consultados, para quienes los modelos de pareja tradicionales han sido transformados por lo político y lo social (Eguiluz, 2007; Tapia, et al., 2009; Garrido, et al., 2008; Arratia, 2009; Tapia, 2001).

En la época contemporánea existen nuevas manifestaciones de ser y formar pareja. Las personas están experimentando diversas formas de establecer vínculos y otras maneras de relacionamiento con el otro. De manera que, así como el concepto de pareja ha cambiado, también lo ha hecho su estructura y su idea de consolidación, que ya no tiene necesariamente como eje principal la permanencia en el tiempo. Arratia (2009) soporta lo anterior, refiriendo que

se inicia una suerte de selección de la pareja, que a su vez considera diversas formas de vincularse, donde el matrimonio como institución no parece del todo necesaria, en la cual los participantes de la pareja tienen diversidad de roles y toman cierta autonomía, que los deja unidos por razones emotivas más que prácticas. Parte entonces el concepto de pareja por amor y con ello también la enorme diversidad de formas de ser y hacer pareja, se puede vivir juntos o separados, tener o no hijos, elegir una pareja heterosexual 


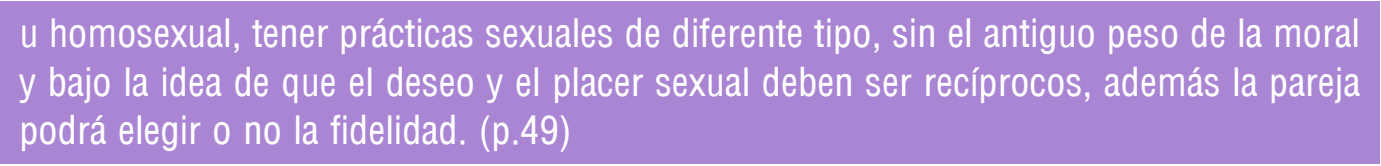

En la actualidad es común identificar expresiones como: poliamor, parejas lat, parejas de segundas nupcias, parejas pluriétnicas, parejas abiertas, amigovios, swinger, entre otros. Esto demuestra que las relaciones humanas tienen la característica de ser cambiantes, variables y dinámicas (Caillé, 2002; De Oliveira, 2011; Bernal, 2013; Inturmendi, 2014; Gatatsopoulo, et al., 2017; Montoya, et al., 2015). De Oliveira (2011) resalta que el actual

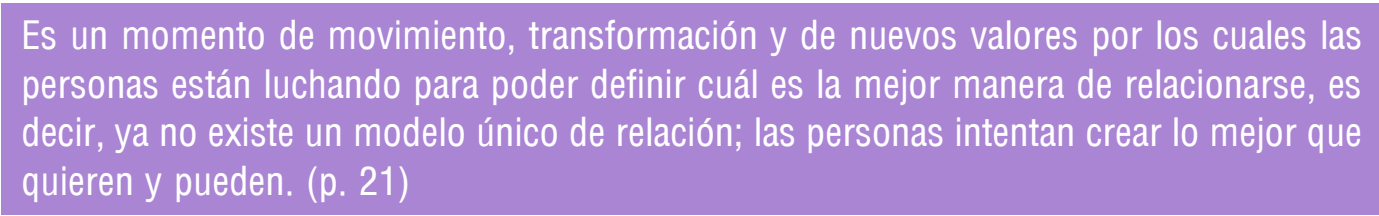

En la siguiente ilustración se sintetiza el cambio de concepción de la pareja a través de la historia, según los autores consultados:

Figura 1

Concepción de la pareja a través de la historia
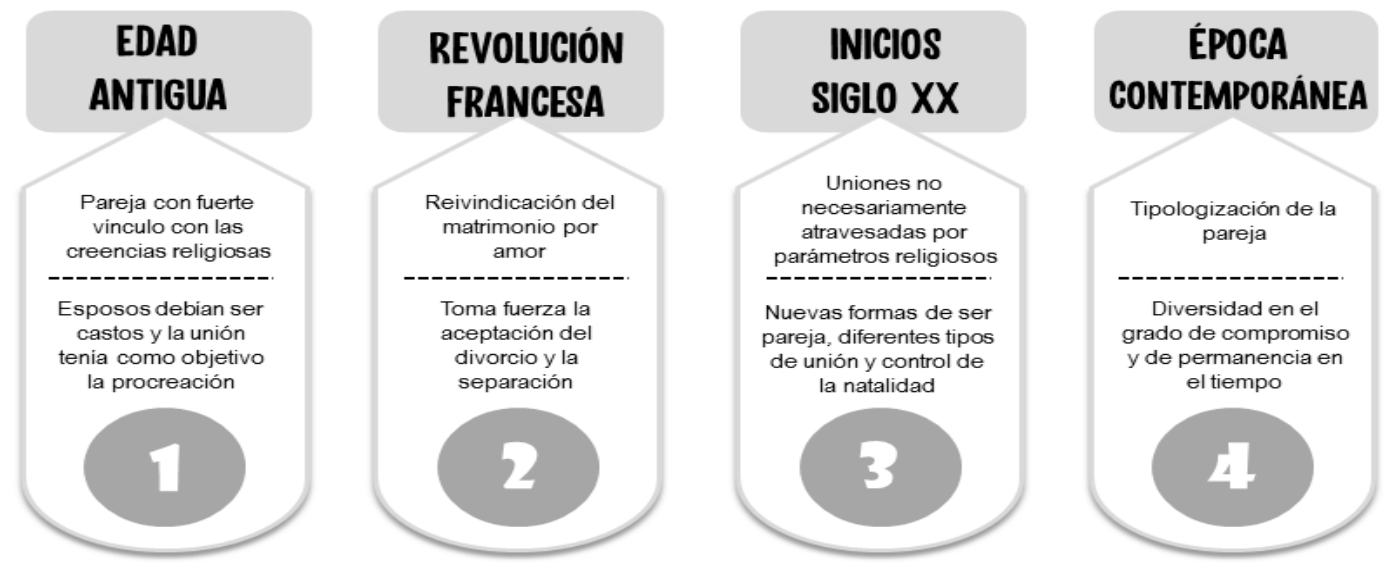

Nota: De Oliveira (2011)

Otro de los hallazgos está relacionado con el concepto de pareja visto como un sistema relacional y circular que contiene estrategias de autorregulación y con dinámicas particulares. Como lo señala Jara (2005),

Desde la teoría sistémica y la terapia familiar se plantea que la pareja es un sistema con mecanismos reguladores propios, con patrones de interacción repetitivos, con comportamientos que dependen uno de otro en forma circular y que como todo sistema tiende hacia la homeostasis. (p. 44) 
Lo anterior, es coherente con la línea de pensamiento de Castrillón (2008), para quien "el proceso y decisión más importante que cada miembro en una pareja realiza es el de seleccionar a una persona con quien va a gastar su existencia y con quien conjuntamente va a estar construyendo una relación" (p. 190).

De esta manera, la pareja como una unidad relacional conforma su propia identidad y emergen en ella patrones de interacción. En este sistema pareja se da la presencia de individualidades que llegan a unirse para formar una vida en común, permeados ambos miembros por sus historias de vida y siendo imperante el todo como más que la suma de las partes (García, 2002; De la Espriella, 2008; Ventura, 2018, Maureira, 2011; Tapia et al., 2009; Garrido et al., 2008; Castrillón, 2008; Pinto, 2018).

Así mismo, desde la perspectiva sistémica se reconoce en la pareja a dos miembros comprometidos en un vínculo amoroso, diferentes y separados por su historia de vida, crianza, familia de origen, valores, cultura, creencias, hábitos, deseos, percepciones, experiencias, aprendizajes, necesidades propias que se unen para aportar a un proyecto en común, generando nuevas necesidades sin desconocer lo ininteligible que puede llegar a ser el otro (Pinto, 2018; De Oliveira, 2011; Fossa et al., 2013; Inturmendi, 2014; Tapia \& Molina, 2014; Pinto, 2005; Garrido et al., 2008; Mora Herrera et al., 2017; Pedroza, 2017; Peñafiel, 2011).

La individualidad referida por los autores puede ser ambivalente, esto es, la persona no quiere establecer raíces emocionales profundas, sino que tiene el deseo de estar sola, pero a su vez acompañada para recorrer un futuro en conjunto guiado por la subjetividad, como lo señala Pedroza (2017):

Los hombres y mujeres de nuestro tiempo están ávidos e incluso desesperados por relacionarse, para sentirse seguros porque saben que son fácilmente descartables, pero a su vez desconfían de estar relacionados y en particular estar relacionados para siempre, porque les tensiona la idea de verse limitados para relacionarse. (pp. 466-467)

Es posible, entonces, identificar que cuando dos individualidades se unen para formar una pareja, es necesario establecer acuerdos para fusionar sus objetivos; estos acuerdos se incorporan en la dinámica de la pareja y es cuando se rompen las reglas establecidas que surgen las problemáticas (García, 2002; Inturmendi, 2014; Ventura, 2018).

De acuerdo con lo hallado en las fuentes, definir la pareja es algo complejo ya que es un concepto inacabado y en constante construcción; las significaciones que se han ido otorgando a la pareja a lo largo del tiempo han evolucionado de acuerdo con las interacciones mediadas por la cultura y por la historia (De Oliveira, 2011; Garrido et al., 2008). 


\section{Habilidades del terapeuta de pareja}

Una segunda categoría producto del análisis de las fuentes consultadas, estuvo relacionada directamente con el terapeuta, pero más que indagar por un rol del mismo dentro de la terapia, se buscaron habilidades 0 destrezas que fueran relevantes durante el proceso terapéutico y que pudieran brindar información pertinente a los profesionales en terapia que se enfrentan al reto de trabajar con parejas.

A partir del rastreo bibliográfico y del ejercicio interpretativo realizado con respecto a esta categoría, se lograron recoger algunas de las habilidades que debe desarrollar un terapeuta de pareja, encontrando que una de las habilidades más salientes tuvo que ver con la perspicacia del terapeuta para profundizar en los motivos de consulta por los cuales la pareja asiste a terapia, seguida de la capacidad de hacer lectura del proceso interaccional de la pareja, así como de la destreza para la construcción de la alianza terapéutica y, finalmente, se recogen algunas características que se pueden integrar y relacionar con lo que se ha nombrado el self del terapeuta, dimensión que engloba la empatía, la neutralidad, la conversación terapéutica, entre otras.

El desarrollo de estas habilidades permite trazar entre la pareja y el terapeuta los objetivos terapéuticos que guiarán el proceso de intervención y crear un ambiente propicio para que ambos miembros de la diada se sientan en un clima de confianza durante las sesiones y encuentros que se establezcan durante el desarrollo de la terapia.

De acuerdo con los autores, dentro de las habilidades que el terapeuta debe desarrollar se encuentra la capacidad de leer el proceso interaccional de la pareja, ya que a partir de esta lectura el terapeuta devela la definición que hace la pareja de su relación, sus acuerdos, sus desacuerdos y la percepción que cada uno de ellos tiene de la situación problema que los convoca a terapia. En las pautas interaccionales se pueden identificar también patrones de comportamiento que generan dolor y se convierten en un círculo de reproche sin salida para la pareja; es así como el terapeuta puede acompañar a los consultantes en la resolución de las situaciones conflictivas, apoyando en la redefinición del problema inicial por el que consultan y generando estrategias que permitan la toma de decisiones frente al proceso (Fossa et al., 2013; De Oliveira 2011; Szmulewicz, 2013; Tapia \& Molina, 2014).

En relación con lo anterior, Tapia y Molina (2014) refieren que

El proceso terapéutico se focaliza en la distinción de pautas interaccionales, atribución de significados y emociones asociadas a esas pautas. El clima emocional encarna 0 corporeiza dichas pautas, las cuales pueden ser percibidas al prestar atención a las vivencias corporales y de naturaleza pre-verbal. Su descripción es usualmente en un lenguaje difuso y ambiguo (el ambiente estaba tenso, funesto, plano). Focalizar en el clima permite orientar el diálogo hacia la mentalización de los cónyuges entre sí y con el terapeuta e introducir complejidad a la perspectiva relacional del problema. ( $p .44)$ 
Dado esto, los autores refieren que para entender el proceso de interacción que se da en las parejas, es necesario que el terapeuta escuche a cada uno de los miembros de la diada, que se genere un diálogo, una conversación en la que cada miembro se sienta escuchado y pueda exponer sus preocupaciones, miedos 0 ansiedades, pero que, a su vez, escuche la narrativa de su pareja. En este sentido, Nieto (2017) dice que

\section{El terapeuta ya no se visualiza como un experto que posee una visión o historia privi- legiada, sino como un facilitador de esta conversación terapéutica, como un maestro 0 una maestra en el arte de la conversación (Goolishian, 1989), es decir que se considerará que cada narrativa y discurso evocado de quienes conforman el sistema terapéutico con- figurarán nuevas versiones de las historia narrada, edificando a partir de allí las nuevas formas de relación en el sistema familiar que resulten funcionales para el mismo. (p. 63)}

A partir de estas narrativas de la pareja, el terapeuta obtiene la información que le permite establecer el motivo de consulta. Los autores refieren que la pareja puede llegar con un motivo de consulta; sin embargo, durante el proceso se puede develar otro motivo que ni la misma pareja tenía claro o había pensado. En este sentido, el terapeuta debe desarrollar la habilidad de buscar información sobre el problema, ampliar y definir el motivo de consulta para dar paso a procesos de reflexividad desde el plano relacional. Su papel es intervenir en las nuevas problemáticas de la pareja contemporánea, proponer soluciones diferentes a las que habitualmente ha intentado la pareja, hacer uso de todos los recursos que están a su alcance, exponer su creatividad, es decir, orientar a la pareja hacia la resignificación del motivo de consulta inicial, mediante un proceso empático, actuando como facilitador y no como experto (Jones \& Asen, 2000; Pinto, 2004; Ventura, 2018; Garzón, 2008; Pinto, 2018; Fossa et al., 2013; Gunther, 2017; Pinto 2000).

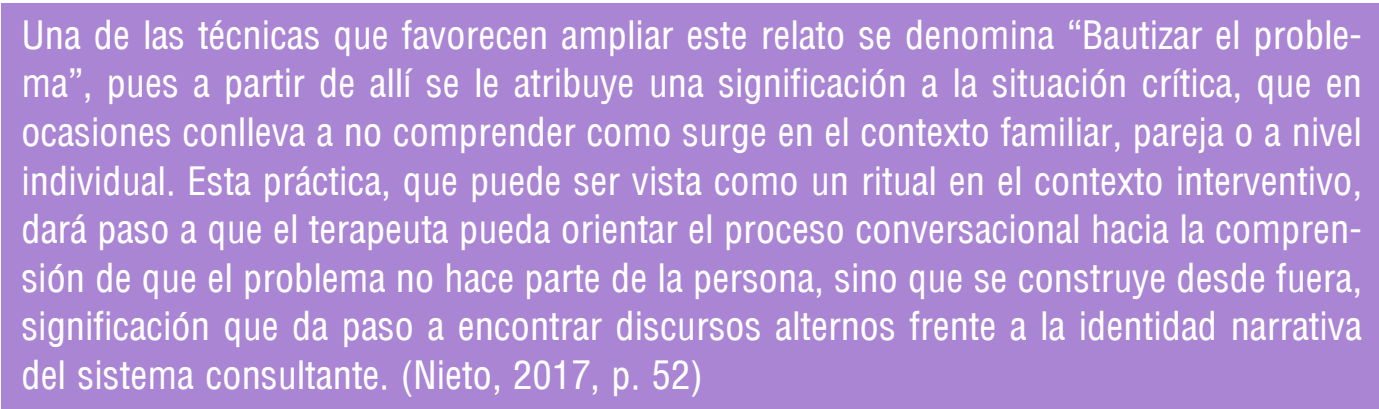

En este proceso de resignificación del motivo de consulta inicial, el terapeuta asume un papel de facilitador, además de una posición neutral; reconoce los esfuerzos de la pareja, crea un ambiente seguro, una comunicación clara, respetuosa y objetiva; propone algunas estrategias de exploración, redefinición, implementación y confrontación, utilizando modelos y técnicas desarrolladas por las distintas escuelas 0 enfoques de terapia familiar.

Al respecto, De la Espriella (2008), afirma que "el terapeuta debe mostrarse neutral, al permitir a ambos participantes la expresión de sus puntos de vista, además de reconocerles los esfuerzos realizados previamente y la asistencia a la terapia" (p. 183). En esta misma vía, Selvini, como se cita en Nieto (2017), expresa que el concepto de neutralidad se entiende como "la postura de los 
terapeutas donde retoman la configuración del problema que hace cada miembro de la pareja, visibilizando la intención de su comunicación, respetando las historias, su autonomía y dando relevancia a todos los significados construidos" (p. 134).

En la medida en que el terapeuta pueda generar un espacio neutral será posible establecer la alianza terapéutica. Es así como otra de las habilidades que se nombra en las diferentes fuentes se refiere al establecimiento de la alianza terapéutica, la cual consiste en "crear un vínculo, co-construir un motivo de consulta relacional y acordar una trayectoria terapéutica" (Tapia \& Molina, 2014, p. 44). En el caso de la terapia de pareja, este vínculo no se construye con cada uno de los individuos, sino con la pareja misma que es el sistema a intervenir, para esto es necesario que el terapeuta desarrolle habilidades como la empatía y la seguridad.

Así mismo, en la alianza terapéutica se establece el objetivo terapéutico y se co-construye un clima terapéutico que permita a sus miembros tener la suficiente confianza en la persona del terapeuta para abordar los problemas importantes para la pareja.

En el establecimiento de esta alianza se devela la importancia de la mirada sistémica, como lo expresa Montesano (2015),

el terapeuta debe prestar mucha atención a otro componente específico de la alianza en el contexto de una TP: la alianza intra-sistema. El terapeuta tiene que ser consciente de que la principal relación que vehiculiza el cambio no es con él o ella, sino la de la propia pareja. Por tanto, el foco terapéutico debe privilegiar la relación de pareja sobre la terapéutica. En esta diferencia radica una de las ventajas principales de la TP: permite trabajar con el contexto en el que se enmarcan los problemas y utilizarlo como fuente de recursos para la intervención. (p.163)

Esto a su vez es reafirmado por Escudero (2009), quien dice que "desde el plano técnico hay una orientación básica: el terapeuta tiene que prestar atención simultánea a las necesidades del sistema, entrelazándolas de una forma que tenga sentido para todos mediante el "reencuadre" del problema" (p. 251).

El conseguir exitosamente esta alianza terapéutica puede representar un aporte significativo para el desarrollo efectivo de la terapia; si este proceso se establece adecuadamente, el terapeuta podrá desarrollar con mayor tranquilidad y fluidez las demás habilidades con las que cuente e implementar las estrategias metodológicas que puedan contribuir a la resignificación del problema al interior de la pareja:

Gracias a la consolidación de una alianza de trabajo entre terapeuta y clientes en esta fase el terapeuta puede sentirse más confiado sobre el uso de técnicas que pueden desencadenar una mayor inseguridad y cambio para los clientes, tales como preguntas de futuro, desafíos, representaciones, redefiniciones y reestructuraciones, uso de historias y metáforas ampliadas, tareas y técnicas no verbales, etc. (Jones \& Asen, 2000, p. 40) 
Finalmente, fue encontrado en el rastreo documental la relevancia del desarrollo del Self del terapeuta, que radica en que el proceso de terapia impacta tanto la vida de los consultantes como la del terapeuta mismo, ya que el terapeuta está inmerso en el intercambio de significados que se construyen en el espacio terapéutico. El conocimiento de sí mismo, la conciencia de las experiencias, sensaciones y emociones que el terapeuta pone en el proceso de terapia, permiten generar un espacio de mayor confianza. Adicionalmente, el reconocimiento propio y del otro posibilita el desarrollo personal y profesional del terapeuta. El self será, entonces, la principal herramienta del terapeuta, aquella que le permite establecer una relación fuerte con la pareja; por tanto, es necesario que el terapeuta se muestre empático, curioso y en negación de un rol de experto.

Al respecto, Szmulewicz (2013) refiere que "el hacer terapia impacta sobre la vida y el quehacer del terapeuta al ser incluido de una manera tan íntima en el mundo interno de los pacientes" (p. 61). Siendo así, el terapeuta deberá tener una capacidad de reflexión como un mecanismo mediante el cual se desarrolla el self, la reflexión entendida como la capacidad de ponerse en el lugar del otro para auto-observarse, salir de sí y convertirse en objeto de reflexión.

En este sentido, plantea Ledesma (2018) que "para explorar el amor en la terapia de pareja es importante que el terapeuta haga una pausa y vea, mantenga una curiosidad genuina por comprender lo que los consultantes traen a sesión" (p. 57). Quiere esto decir que el terapeuta debe ser empático, ya que la empatía permite ver a través de los ojos del otro; como lo refieren Tapia y Molina (2014) "La empatía está inserta en la mutualidad de una interacción regulatoria dentro del sistema terapéutico, son procesos concurrentes y recíprocos. Es una influencia mutua que transcurre como micro proceso, a menudo oculto tras el umbral de la conciencia" (p. 44).

\section{Discusión}

De acuerdo con las dos categorías que fueron trazadas como ejes principales de análisis en esta investigación, podemos poner como centro de discusión de este trabajo, que conceptualizar a la pareja es un ejercicio complejo, debido a que ha sido un concepto modificado, interpretado y adoptado, desde diferentes dimensiones sociales; es por esto, que no se puede concebir a la pareja humana de una manera estática, estable 0 inamovible, ya que esta está marcada por parámetros sociales, religiosos y políticos (De Oliveira, 2011; Garrido et al., 2008; Jaramillo et al., 2016).

Es así como la mirada del terapeuta de pareja, con respecto a cómo desde su praxis entiende, conoce e interpreta a la pareja, lo situará ante una intervención terapéutica sin sesgos o prejuicios. Aquí, la segunda categoría de esta investigación, entrega algunas de las habilidades que el terapeuta debe desarrollar, y que han sido develadas en las intervenciones y sesiones con parejas, entre las que se destaca la importancia de identificar el motivo de consulta, y la lectura del proceso interaccional, exaltando la necesidad de profundizar en las narrativas del sistema consultante desde la circularidad. 
En cuanto al proceso de investigación, al ser esta de índole documental, representa una limitación para las investigadoras, ampliar la información desde un enfoque más práctico y experiencial, sin embargo, se identifica que, si bien se dan diferentes interpretaciones y conceptos de pareja, se observa insuficiente profundización en aspectos emocionales y relacionales que trae consigo la interacción de las diversas tipologías de pareja.

En esta misma línea, en los hallazgos se observa que las habilidades del terapeuta de pareja están directamente relacionadas con las desarrolladas por los terapeutas de familia; sin embargo, como bien es sabido hay diferencias sustanciales entre estos dos sistemas (el familiar y el de pareja) y por esto se haría significativo profundizar y hacer una construcción teórica tanto del rol del terapeuta de pareja como de sus diferentes herramientas para intervenir la diada.

Finalmente, se encuentra que si bien existe una cantidad significativa de bibliografía relacionada con el tema de terapia de pareja, la mayor parte de esta se encuentra en idioma extranjero, por lo cual, se evidencia la necesidad de realizar más avances investigativos desde los países latinoamericanos y que sean compartidos con la comunidad académica, fortaleciendo así el conocimiento y la producción intelectual en estos temas, que permitan establecer una mirada más amplia de la pareja, que como ya se ha dicho, ha demostrado ser cambiante por excelencia y nos demuestra que no existe un patrón único de relación entre ellas.

\section{Conclusiones}

Después de realizar la revisión documental, a manera de conclusiones se presentan los siguientes aspectos. Respecto a las categorías de análisis, fue posible identificar que el concepto de pareja, se constituye por diversos factores como la cultura, la historia, las creencias y el contexto; por tanto, este concepto se deriva ciertamente de una construcción social. Los cambios históricos y culturales que se han dado en la sociedad han influido indudablemente en que las parejas presenten nuevas formas de nominarse y constituirse. Es así que en el análisis documental fue posible ver el tránsito del estado civil de las parejas, la aparición de nuevas tipologías y los nuevos acuerdos al interior de las parejas que determinan su grado de compromiso y su permanencia en el tiempo.

Por otro lado, y teniendo en cuenta la tendencia actual de las familias y las parejas a buscar ayuda terapéutica, el rol del terapeuta ha tomado mayor importancia, lo cual implica el desarrollo de competencias no solo teóricas sino también técnicas y personales entre las que se encuentran habilidades como definición del motivo de consulta, capacidad de hacer lectura del sistema consultante, construcción de la alianza terapéutica y el desarrollo del self reconociendo que el terapeuta en sí mismo es objeto de cuidado en el proceso terapéutico. 
Finalmente, el reto para próximas investigaciones será profundizar en las nuevas expresiones de pareja, el vínculo amoroso, el vínculo erótico, los tipos de amor que han ido surgiendo y surgirán en el tiempo, los cuales darán al campo disciplinario una gama de conocimientos teóricos, conceptuales y de intervención para el abordaje sistémico de la pareja. Así mismo, se hace necesario en futuros estudios, establecer con mayor ahínco las características y habilidades que debe desarrollar un terapeuta de pareja, pues si bien se habla ampliamente en la literatura sobre la figura del psicoterapeuta, se requiere mayor producción intelectual e investigativa en el campo de la atención a parejas.

\section{Conflicto de intereses}

Las autoras declaran la inexistencia de conflicto de interés con institución o asociación comercial de cualquier índole.

\section{Referencias}

Agudelo Bedoya, M. E., \& Palacio Correa, M. A. (2015). Problemáticas en la relación de pareja y de padres e hijos de los consultantes del Centro de Familia de la UPB. Revista de la Facultad de Trabajo Social, 31(31), 75-92. https://revistas.upb.edu.co/index.php/trabajosocial/article/ view/2401

Arratia, E. (2009). Terapia sexual en pareja desde la perspectiva constructivista cognitivo [Tesis de Maestría, Universidad de Chile]. Repositorio Académico de la Universidad de Chile. http://repositorio.uchile.cl/bitstream/handle/2250/105783/cs-arratia_e.pdf?sequence $=3 \&$ isAllowed $=$ y

Bernal, I. (2013). “Juntos pero separados”. Parejas LAT en la ciudad de Medellín. Revista de la Facultad de Trabajo Social, 29(29), 173-194. https://revistas.upb.edu.co/index.php/trabajosocial/article/ download/2468/2243/4391

Caillé, P. (2002). El destino de las parejas: avatares y metamorfosis de la pasión. Redes, 10, 9-18.

Castrillón, E. (2008). Terapia de pareja: una mirada a sus procesos. Revista Colombiana de Psiquiatría, 37(1), 187-197. https://www.redalyc.org/pdf/806/80615420015.pdf

De la Espriella, R. (2008). Terapia de pareja: abordaje sistémico. Revista Colombiana de Psiquiatría, 37(1), 175-186. https://www.redalyc.org/pdf/806/80615420014.pdf 
De Oliveira, V. R. (2011). Estudio cualitativo caracterización de las relaciones conyugales en los momentos de crisis previos a la separación o divorcio. [Tesis Doctoral, Universidad de Deusto]. Base de datos de Tesis Doctorales (TESEO). https://www.educacion.gob.es/teseo/imprimirFicheroTesis.do?idFichero $=2$ YetRwCOHtw\%3D

Eguiluz, L. (2007). Historia de la pareja humana. En L. Eguiluz (Comp.), Entendiendo a la pareja (pp. 1-18). Pax.

Escudero, V. (2009). La creación de la alianza terapéutica en la terapia familiar. Apuntes de psicología, 27(2-3), 247-259. http://www.apuntesdepsicologia.es/index.php/revista/article/view/145

Estrada, P., López, R. D., \& Posada, F. A. (1997). Cibernética de segundo orden y abordaje a la familia en la Universidad Pontificia Bolivariana. Revista de la Facultad de Trabajo Social de la Universidad Pontificia Bolivariana, 14, 30-47. https://bit.ly/3qvFhYM

Fossa, P., Benavente, G., \& Pimentel, V. (2013). Construcción del foco de intervención en primera sesión de terapia de pareja: Un análisis de proceso. De Familias y Terapias, (34), 119-135. https://bit.ly/3F8W7k9

García, J. (2002). La estructura de la pareja: implicaciones para la terapia cognitivo conductual. Ciencia y Salud, 13(1), 89-125. https://www.redalyc.org/pdf/1806/180618083004.pdf

Garrido, A., Reyes, A., Torres, L. E., \& Ortega, P. (2008). Importancia de las expectativas de pareja en la dinámica familiar. Enseñanza e Investigación Psicológica, 13(2), 231-238. https://www. redalyc.org/pdf/292/29213203.pdf

Garzón, D. (2008). Autorreferencia y estilo terapéutico: su intersección en la formación de terapeutas sistémicos. Diversitas: Perspectivas en Psicología, 4(1), 159-171. https://revistas.usantotomas. edu.co/index.php/diversitas/article/view/274

Gatatsopoulo, E., Sánchez, F., \& Pérez, M. D. (2017). La pareja reconstituida: un desafío para los terapeutas de pareja. Redes, 37, 87-100. https://redesdigital.com.mx/index.php/redes/article/ view/233

Gunther, C. (2017). La alianza terapéutica en terapia familiar con progenitores separados en conflicto. Un modelo del proceso de cambio intrasistema [Tesis doctoral, Universitat Ramon Llull]. Tesis doctorales en Xarxa. https://www.tdx.cat/bitstream/handle/10803/403375/ TD_G\%C3\%BCnther_2017.pdf?sequence $=1 \&$ isAllowed $=y$

Inturmendi, A. B., (2014). Un viaje transgeneracional a través del vínculo de pareja. Escuela de Vasco Navarra de Terapia Familiar y de Pareja. https://www.avntf-evntf.com/wp-content/ uploads/2016/11/Iturmendi-A.B.-Trab.-3\%C2\%BA-online-13.pdf

Jara, C. (2005). Tipos de parejas y objetivos terapéuticos. De Familias y Terapias (20), 43-49. https://docplayer.es/9766775-Tipos-de-parejas-y-objetivos-terapeuticos-1.html

Jones, E., \& Asen, E. (2000). Terapia sistémica de pareja y depresión. Desclée de Brouwer. 
Mora Herrera, Y., Recalde David, M. V., Montoya Vertel, Y. A., González Herrera, M. P., Paternina Montiel, D. I., Rendón Botero, M. L., López Sepúlveda, P. A., Rodriguez Goez, L. A., Yepes Marulanda, S., Vélez Montoya, R. D., Hernández Moreno, L. V., Sánchez Peláez \& R. A., Bedoya Cardona, L. M. (2017). Reflexiones sobre la ética del psicólogo. Poiésis, (33), 59-74. DOl: https://doi.org/10.21501/16920945.2496

Ledesma, A. (2018). Reflexiones entre el amor romántico, el amor maduro, la locura y el amor sin etiqueta en psicoterapia de pareja. Redes, 37, 51-60. https://redesdigital.com.mx/index.php/ redes/article/view/232/0

Maureira, F. (2011). Los cuatro componentes de la relación de pareja. Revista Electrónica de Psicología Iztacala, 14(1), 321-322. http://www.revistas.unam.mx/index.php/repi/article/ view/24815

Minuchin, S., \& Fishman, C., (2004). Técnicas de terapia Familiar. Paidós.

Montesano, A. (2015). Claves fundamentales en terapia de pareja: Una guía de navegación para la práctica. Revista de Psicoterapia, 26(102), 161-174.

Montoya, L., Ocampo, L. N., Rodríguez, A., \& Giraldo, C. M. (2015). Tú en tu casa, yo en la mía: Parejas LAT. Poiésis, (30), 178-183. https://www.funlam.edu.co/revistas/index.php/poiesis/ article/view/1860

Ríos Madrid, M. (2017). Reflexiones sobre la ética del psicólogo. Poiésis, (33), 59-74. https://doi. org/10.21501/16920945.2496

Nieto, P. (2017). Una mirada terapéutica a los problemas de pareja recién constituida [Tesis de Maestría, Universidad Santo Tomás de Aquino]. Repositorio Institucional de la Universidad Santo Tomás. https://repository.usta.edu.co/bitstream/handle/11634/10303/ Nietomar\%C3\%ADa2018.pdf?sequence $=1 \&$ isAllowed $=y$

Pedroza, R. (2017). Los cambios del vínculo amoroso en la posmodernidad. Revista Electrónica de Psicología Iztacala, 20(2), 464-478. http://www.revistas.unam.mx/index.php/repi/article/ view/60810

Peñafiel, 0. (2011). Ruptura amorosa y terapia narrativa. Ajayu Órgano de Difusión Científica del Departamento de Psicología UCBSP, 9(1), 53-86. http://www.scielo.org.bo/scielo. php?pid=S2077-21612011000100002\&script =sci_abstract

Pinto, B. (2000). Terapia de pareja: una perspectiva cognitiva-sistémica. Revista Ciencia y Cultura, $8,79-86$.

Pinto, B. (2004). La psicoterapia relacional sistémica y el psicoterapeuta. Ajayu Órgano de Difusión Científica del Departamento de Psicología UCBSP, 2(2), 17-34. 
Pinto, B. (2005) Colisión, colusión y complementariedad en las relaciones conyugales. Ajayu Órgano de Difusión Científica del Departamento de Psicología UCBSP, 3(1), 1-29. https://www.ucb. edu.bo/publicaciones/ajayu/v3n1/v3n1.htm

Pinto, B. (2018). Teoría triangular del amor y teoría del compromiso en la psicoterapia de pareja. Redes, 37, 37-50. 2018. http://www.redesdigital.com.mx/index.php/redes/article/view/225

Szmulewicz, T. (2013). La persona del terapeuta: eje fundamental de todo proceso terapéutico. Revista Chilena de Neuro-psiquiatría, 51(1), 61-69. https://dx.doi.org/10.4067/ S0717-92272013000100008

Tapia, L., \& Molina, M. (2014). Primera entrevista en terapia de pareja: co-construcción de un encuentro situado. Revista Chilena de Neuropsiquiatría, 52(1), 42-52.

Tapia, L. (2001). Algunas consideraciones para una terapia de pareja basada en la evidencia. De Familias y Terapias, 9(14-15), 7-30. http://cesch.cl/wp-content/uploads/2013/05/TerapiaParejaEvidencia.pdf

Tapia, L., Poulsen, G., Armijo, A., Pereira X., \& Sotomayor, P. (2009). Resolución de entrampes en parejas en conflicto: aproximaciones desde las parejas y los terapeutas. Revista Argentina de Psicología Clínica, 18(2), 101-114. http://www.cienciared.com.ar/ra/usr/35/803/racp_xviii_2_ pp101_114.pdf

Ventura, D. (2018). Infidelidad en las relaciones de pareja: Algunas consideraciones para su abordaje en terapia. Redes, 37, 51-60. http://www.redesdigital.com.mx/index.php/redes/ article/view/232

Vicencio, J. (2011). Mapas del amor y la terapia de pareja. Pax México. 